\title{
MRS Proceedings
}

http://journals.cambridge.org/OPL

Additional services for MRS Proceedings:

Email alerts: $\underline{\text { Click here }}$

Subscriptions: Click here

Commercial reprints: $\underline{\text { Click here }}$

Terms of use : $\underline{\text { Click here }}$

\section{Effect of Oxygen Pressure on the Initial Oxidation Behavior of $\mathrm{Cu}$ and $\mathrm{Cu}-\mathrm{Au}$ Alloys}

Langli Luo, Yihong Kang, Zhenyu Liu, Judith C Yang and Guangwen Zhou

MRS Proceedings / Volume 1318 / 2011

DOI: 10.1557/opl.2011.920

Link to this article: http://journals.cambridge.org/abstract_S1946427411009201

How to cite this article:

Langli Luo, Yihong Kang, Zhenyu Liu, Judith C Yang and Guangwen Zhou (2011).

Effect of Oxygen Pressure on the Initial Oxidation Behavior of $\mathrm{Cu}$ and $\mathrm{Cu}-\mathrm{Au}$ Alloys. MRS Proceedings,1318, mrsf10-1318-ss07-06 doi:10.1557/opl.2011.920

Request Permissions : $\underline{\text { Click here }}$ 


\title{
Effect of Oxygen Pressure on the Initial Oxidation Behavior of $\mathrm{Cu}$ and $\mathrm{Cu}-\mathrm{Au}$ Alloys
}

\author{
Langli Luo ${ }^{1}$, Yihong Kang ${ }^{2}$, Zhenyu Liu ${ }^{2}$, Judith C Yang ${ }^{2}$, Guangwen Zhou ${ }^{1}$ \\ ${ }^{1}$ Department of Mechanical Engineering \& Multidisciplinary Program in Materials Science and \\ Engineering, State University of New York, Binghamton, NY 13902 \\ ${ }^{2}$ Department of Mechanical Engineering and Materials Science, University of Pittsburgh, \\ Pittsburgh, PA 15261
}

\begin{abstract}
A wide information gap exists between our present atomic-scale knowledge of metal oxidation derived from conventional ultrahigh vacuum (UHV) surface science experiments and the oxidation mechanisms obtained from the growth of bulk oxide thin films under technologically relevant realistic (or near-) atmospheric conditions. To bridge this pressure gap, we present an in-situ transmission electron microscopy (TEM) study of the initial oxidation stage of $\mathrm{Cu}(100)$ and $\mathrm{Cu}-\mathrm{Au}(100)$ surfaces where the oxygen partial pressure varies from $5 \times 10^{-4}$ to 150 Torr. For $\mathrm{Cu}(100)$, with increasing oxygen partial pressure $\left(\mathrm{pO}_{2}\right)$, the nucleation density of the oxide islands increases and so does the growth rate of the oxide islands. As the $\mathrm{pO}_{2}$ continues to increase, a transition from epitaxial cube-on-cube $\mathrm{Cu}_{2} \mathrm{O}$ islands to randomly oriented oxide islands is observed. A kinetic model based on the classic heterogeneous nucleation theory is developed to explain the effect of oxygen partial pressure on the oxide orientation. It is shown that such a transition in the oxide nucleation orientation is related to the effect of oxygen pressure on the nucleation barrier and atom collision rate. The $\mathrm{Cu}-\mathrm{Au}(111)$ alloy revealed the same oxygen pressure dependency of the oxide nucleation orientation as pure $\mathrm{Cu}$ oxidation.
\end{abstract}

\section{INTRODUCTION}

Fundamental understanding of metal and alloy oxidation is of significant importance to a wide range of applications including corrosion, catalysis, fuel reactions, electrochemistry, and thin film processing. It is well known that metal oxidation involves hierarchical multiple length scales and proceeds generally from oxygen surface chemisorptions to oxide nucleation and growth and then to the formation of a continuous, macroscopically thick oxide layer. Over the past decades, surface science studies on the oxidation of metal surfaces under idealized conditions, i.e., ultrahigh vacuum (UHV), have gathered a wealth of knowledge including the surface structure, adsorption sites, and reaction pathways. However, the resulting profound understanding of the reaction mechanism obtained under rarefied UHV conditions does not automatically translate into an equally good understanding of the technologically relevant realistic (or near-) atmospheric oxidation. For example, it has been shown recently that the surface oxidation proceed readily under high pressure oxidation conditions, but appear not to proceed under the low pressure conditions typical of a vacuum experiment, despite favorable thermodynamics[1-5]. Therefore, it is paramount to bridge this pressure gap by determining the difference in the response of a metal surface exposed to low and high pressures of oxygen gas.

We have utilized in situ TEM techniques to study the initial stages of $\mathrm{Cu}$ and $\mathrm{Cu}-\mathrm{Au}$ oxidation for bridging the information gap between the knowledge obtained from surface science methods which mainly focus on the adsorption of up to a few oxygen layers and that of traditional oxidation theories of bulk oxide growth [6-10]. In this work, we particularly examine 
how the difference in oxygen pressure can lead to different behaviors of oxide nucleation during the initial oxidation of $\mathrm{Cu}$ surfaces. We have extended our experimental system from pure $\mathrm{Cu}$ to $\mathrm{Cu}-\mathrm{Au}$ alloys. Alloying can affect the nucleation and growth of early stage of oxide islands and then influence the microstructures of the oxide layer as well as the oxidation resistance properties. By alloying $\mathrm{Cu}$ with $\mathrm{Au}$, the oxidation behavior can be changed both thermodynamically and kinetically in ways of altering surface and interface energies, preferred adsorption sites and diffusion paths.

\section{EXPERIMENT}

Our in situ oxidation experiments were carried out in a modified JEOL 200CX TEM. This microscope is operated at $200 \mathrm{keV}$ and equipped with a UHV chamber with a base pressure $\sim 10^{-8}$ Torr. A controlled leak valve is connected to the column of the microscope permits introduction of gases directly into the microscope. Single crystal $\sim 1000 \AA$ of $\mathrm{Cu}$ and $\mathrm{Cu}-\mathrm{Au}$ alloy thin films were grow on $\mathrm{NaCl}$ substrates epitaxially by $\mathrm{UHV}$ e-beam evaporator with a base pressure $\sim 10^{-9}$ Torr. The films were removed from the $\mathrm{NaCl}$ substrate by flotation in deionized water, washed and mounted on a specially designed TEM sample holder, which allows for resistive heating at temperatures between room temperature and $1000^{\circ} \mathrm{C}$. The native $\mathrm{Cu}$ oxide is removed by annealing the alloy films in the TEM under vacuum conditions at $\sim 750^{\circ} \mathrm{C}$ or by in situ annealing in hydrogen gas at a pressure of $5 \times 10^{-5}$ Torr but at a lower temperature $\sim 350^{\circ} \mathrm{C}$ resulting in a clean metal surface. Oxygen gas of $99.999 \%$ purity can be admitted into the column of the microscope through the leak valve at a partial pressure $p \mathrm{O}_{2}$ between $5 \times 10^{-4}$ and 760 Torr for $30 \mathrm{mins}$. For oxidation under $p \mathrm{O}_{2}>1 \times 10^{-4}$ Torr, the chamber is isolated in order to protect microscope and the in situ observation can be made when $p \mathrm{O}_{2}<1 \times 10^{-4}$ Torr.

\section{RESULTS AND DISCUSSION}

Extensive in situ TEM oxidation studies have been carried out on $\mathrm{Cu}(100)$ and $\mathrm{Cu}-$ $\mathrm{Au}(111)$ surfaces to elucidate the correlation between oxygen partial pressure and the oxide growth morphologies. Fig. 1 shows the results of in situ bright-field TEM images of $\mathrm{Cu}(100)$ surfaces oxidized at $350^{\circ} \mathrm{C}$ and different $\mathrm{pO}_{2}$ for 30 mins. It shows that the nucleation density of $\mathrm{Cu}_{2} \mathrm{O}$ islands increases with increasing the oxygen partial pressure from $\mathrm{pO}_{2}=5 \times 10^{-4}$ to 50 Torr. The selected-area electron diffraction (SAED) patterns from the oxidized surfaces reveal that epitaxial $\mathrm{Cu}_{2} \mathrm{O}$ islands (i.e., the cube-on-cube epitaxy) are nucleated under the lower $\mathrm{pO}_{2}$ ( $<5$ Torr). It can be seen that the diffraction spots from $\mathrm{Cu}_{2} \mathrm{O}$ islands are aligned well with that of $\mathrm{Cu}(100)$ surface. From $\mathrm{pO}_{2}=5$ Torr, the pattern began to show double diffraction spots from both oxides and $\mathrm{Cu}$ surface since oxide islands covered most area of $\mathrm{Cu}$ surface. The double diffraction becomes more notable under $\mathrm{pO}_{2}=50 \mathrm{Torr}$, where the oxide islands are coalesced to form a continuous oxide layer and the intensity of diffraction spots from continuous oxide film is stronger than that of the $\mathrm{Cu}$ substrate layer. 


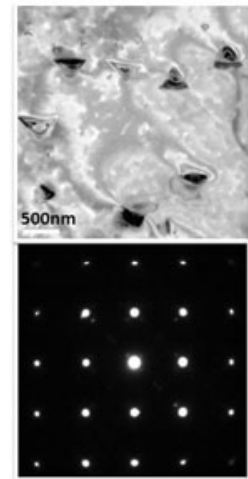

$\mathrm{P}\left(\mathrm{O}_{2}\right)=5 \times 10^{-4}$ Torr
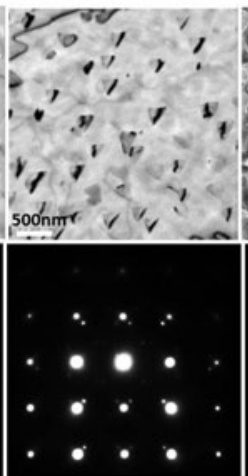

$\mathrm{P}\left(\mathrm{O}_{2}\right)=5 \times 10^{-2} \mathrm{Torr}$
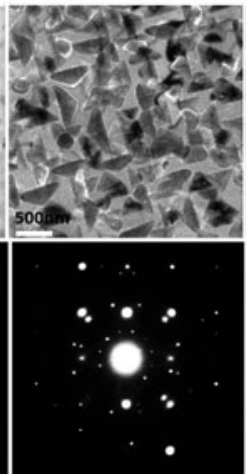

$\mathrm{P}\left(\mathrm{O}_{2}\right)=5$ Torr
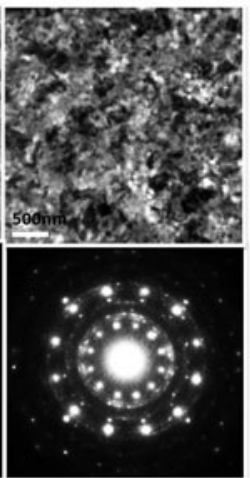

$\mathrm{P}\left(\mathrm{O}_{2}\right)=50$ Torr

Fig. 1 The bright-field TEM images and SAED patterns of $\mathrm{Cu}(100)$ surface oxidized at $350^{\circ} \mathrm{C}$ for 30 mins with oxygen partial pressures from $5 \times 10^{-4}$ to 50 Torr

The above observation indicates that the oxide remained the epitaxial growth with the $\mathrm{Cu}$ substrate from the stages of nucleating oxide islands to the growth of the continuous $\mathrm{Cu} 2 \mathrm{O}$ layer for the oxygen pressures up to 50 Torr. However, randomly oriented oxide islands are observed to nucleate for the oxidation at $\mathrm{pO}_{2}=150$ Torr and above. Fig. 2 shows the oxidation at the different oxygen pressures, the presence of $\mathrm{Cu}_{2} \mathrm{O}$ diffraction rings seen in Fig.2(c) reveals the randomly orientation of the oxide islands.
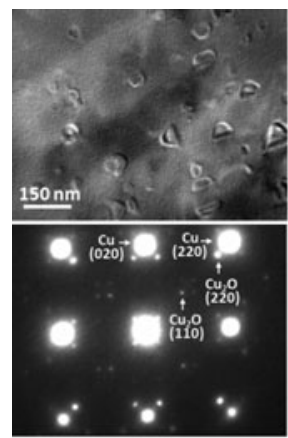

(a)

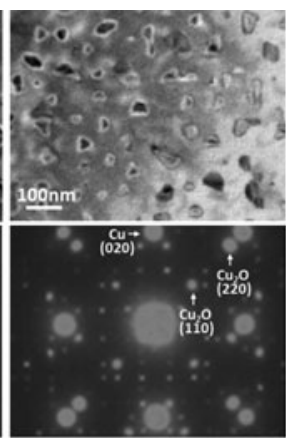

(b)

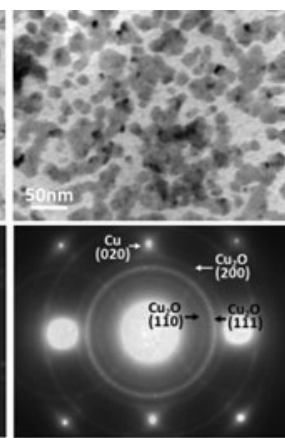

(c)

Fig. 2 (Upper panel) The bright-field TEM images of $\mathrm{Cu}_{2} \mathrm{O}$ islands formed $\mathrm{Cu}(100)$ oxidized at $350^{\circ} \mathrm{C}$ and different oxygen pressures, (a) $\mathrm{pO}_{2}=5 \times 10^{-4}$ Torr, (b) $\mathrm{pO}_{2}=0.5$ Torr, and (c) $\mathrm{pO}_{2}=$ 150 Torr; (Lower panel) SAED patterns from the corresponding oxidized $\mathrm{Cu}(100)$ surfaces

Figure 3 shows the high-resolution (HR) TEM images of the oxidized $\mathrm{Cu}$ surfaces, which further confirm this pressure-dependent orientation of oxide nuclei. Figure 3(a) is a [001] zoneaxis HRTEM image obtained from the $\mathrm{Cu}$ surface oxidized under $\mathrm{pO}_{2}=0.5$ Torr, where the two- 
dimensional (2D) moire fringe pattern caused by the overlapping of $\mathrm{Cu}_{2} \mathrm{O}$ and $\mathrm{Cu}$ lattices is visible. The moiré fringes running parallel to $\{101\}$ lattice planes of the $\mathrm{Cu}$ substrate suggest the $\mathrm{Cu}_{2} \mathrm{O}$ islands have the cube-on-cube epitaxy with the $\mathrm{Cu}$ lattices, i.e., the equivalent planes and directions of $\mathrm{Cu}_{2} \mathrm{O}$ islands and the $\mathrm{Cu}$ substrate are matched. Figure 3(b) is a [001] zone axis HRTEM image of the $\mathrm{Cu}(100)$ surface oxidized under $\mathrm{pO}_{2}=150$ Torr, where the non epitaxial nucleation of $\mathrm{Cu}_{2} \mathrm{O}$ islands can be evidenced by the absence of strong $2 \mathrm{D}$ moiré fringe contrast and the inset Fourier transform pattern of the HRTEM image.

The results indicate that the epitaxial nucleation of oxide islands cannot be maintained within the whole range of oxygen pressures. A kinetic model [11] based on the effect of oxygen pressure on the oxide nucleation barrier and atom collision rate has been developed to account for this transition of oxide nucleation orientation. The oxide nucleation rate $J$ is obtained as

$$
\frac{J}{B}=J_{0} \times \exp \left(-\frac{16 \pi \Omega^{2}(2+n)(1-n)^{2}}{3(k T)^{3}\left[\ln \left(\frac{p O_{2}}{p O_{2}^{e}}\right)\right]^{2}} \sigma_{N o}^{3}\right)
$$

with $J_{0}=\left[\frac{4(1+n)}{(2+n)(1-n)}\right]^{1 / 2} \times\left(\frac{p O_{2}}{p O_{2}^{e}}\right) \times \ln \left(\frac{p O_{2}}{p O_{2}^{e}}\right)$

$\mathrm{n}$ depends on the interaction and structural match between the oxide nucleus and the metal substrate, and is related to the interfacial tension between the oxide and metal substrate by $n=\left(\sigma_{S O}-\sigma_{N S}\right) / \sigma_{N O}$

where $\sigma_{\mathrm{NS}}$ and $\sigma_{\mathrm{SO}}$ are the interfacial free energies between the oxide nucleus and metal substrate and the substrate and oxygen gas, respectively. $\Omega$ is the volume of oxygen atoms in the oxide phase; $\sigma_{\mathrm{NO}}$ denotes the specific interfacial energy between oxide nucleus and oxygen gas; $\mathrm{k}$ and $\mathrm{B}$ are constants for a certain species(e.g. $\mathrm{Cu}$ ); $\mathrm{pO}_{2}$ the actual oxygen pressure during the oxidation, $p O_{2}^{e}$ the equilibrium oxygen pressure as given by the Ellingham diagram for most metal oxides[12].

The change in the nucleation orientation under different oxygen pressures can be physically understood as follows. At low oxygen pressure, the nucleation barrier is very high, and the top priority to accelerate the nucleation kinetics is to lower the nucleation barrier. Therefore, heterogeneous nucleation with the strong interaction and good structural match between an oxide island and the metal substrate will be kinetically favored. Conversely, at high oxygen pressures, the nucleation barrier is reduced and the exponential term becomes less important. Instead, the issue of effective collisions, described by the collisional pre-factor, becomes important. The nucleation of oxide islands with weak interaction and poor structural match with the metal substrate is enhanced. Kinetically speaking, in order to obtain the epitaxial oxide film on the metal substrate by oxidation, the oxygen pressure should be relatively low. If the oxygen pressure is too high, the kinetics leads to a deviation of nucleating oxide islands from the orientation of the metal substrate and the epitaxial relation will be lost. 

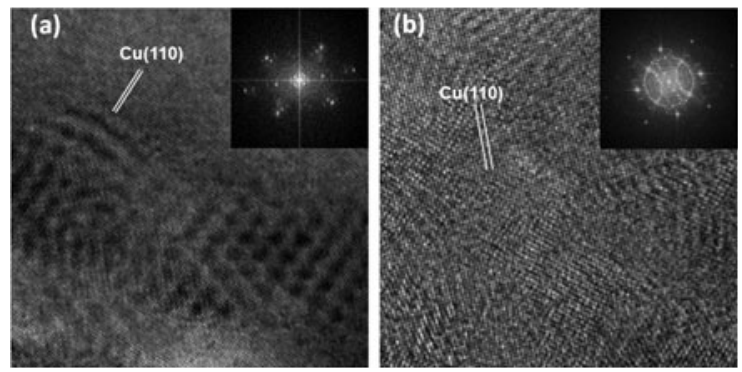

Fig. 3 HRTEM images of $\mathrm{Cu}(100)$ oxidized under different oxygen pressures, (a) $\mathrm{pO}_{2}=0.5$ Torr and (b) $\mathrm{pO}_{2}=150$ Torr. The strong $2 \mathrm{D}$ moiré fringe pattern implies the epitaxial nucleation of $\mathrm{Cu}_{2} \mathrm{O}$ islands; the absence of 2D moire fringes in (b) suggests the non epitaxial nucleation of $\mathrm{Cu}_{2} \mathrm{O}$ islands on the surface. Inset is the Fourier transform of the HRTEM images, which reveal the similar diffraction patterns as those in Figure 2.

We then extended the various oxygen pressure experiments to $\mathrm{Cu}-\mathrm{Au}$ system and examined nucleation orientations of oxide islands on $\mathrm{Cu}-\mathrm{Au}(111)$ surfaces under oxygen partial pressure ranging from $5 \times 10^{-4}$ Torr to 500Torr. Fig. 4 shows the bright field TEM images and correlated SAED patterns from the oxidation under the different oxygen pressures. Similar as $\mathrm{Cu}$ (100), the epitaxial growth of $\mathrm{Cu}_{2} \mathrm{O}$ islands were maintained at lower oxygen pressure $\left(\mathrm{pO}_{2}<\right.$ 5Torr); the diffraction pattern starts to show deviation from the expitaxy of $\mathrm{Cu}-\mathrm{Au}$ (111) surface in the medium oxygen pressure range. The selected-area electron diffraction (SAED) patterns from the oxidized surfaces reveal that randomly oriented oxide islands are nucleated for the oxidation at $\mathrm{pO}_{2}=150$ Torr and above, as revealed by presence of $\mathrm{Cu}_{2} \mathrm{O}$ diffraction rings.

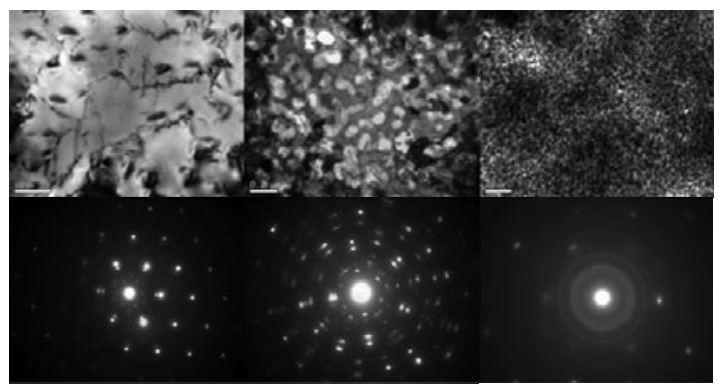

(A)

(B)

(C)

Fig. $4 \mathrm{Cu}-\mathrm{Au}(111)$ surface oxidized at $350^{\circ} \mathrm{C}$ for 30 mins at $\mathrm{pO}_{2}=\mathrm{A}$ ) 0.005 Torr; B)5Torr; C) 150 Torr. 


\section{CONCLUSIONS}

In conclusion, the effect of oxygen pressure on the nucleation and growth of oxide islands during initial oxidation of $\mathrm{Cu}(100)$ and $\mathrm{Cu}-\mathrm{Au}(111)$ is examined. The increase of oxygen partial pressure increases the density of oxide islands. The oxidation under low oxygen pressures leads to epitaxial oxide islands while increasing oxygen pressure results in randomly oriented oxide nuclei. It is shown that such a nucleation orientation transition is caused by the opposite effect (i.e., epitaxial vs. non epitiaxial) of oxygen pressure on the nucleation barrier and atom collision rates.

\section{ACKNOWLEDGMENTS}

This research is financially supported by the U.S. Department of Energy, Office of Basic Energy Sciences, Division of Materials Sciences and Engineering under Award No. DE-FG0209ER46600.

\section{REFERENCES}

1. E. Lundgren, J. Gustafson, A. Mikkelsen, J. N. Andersen, A. Stierle, H. Dosch, M. Todorova, J. Rogal, K. Reuter, and M. Scheffler, Phys. Rev. Lett. 92, 046101 (2004).

2. J. A. Eastman, P. H. Fuss, L. E. Rehn, P. M. Baldo, G. W. Zhou, D. D. Fong, and L. J. Thompson, Appl. Phys. Lett. 87, 051914 (2005).

3. K. Lahtonen, M. Hirsimaki, M. Lampimaki, and M. Valden, The Journal of Chemical Physics 129, 124703 (2008).

4. I. Lyubinetsky, S. Thevuthasan, D. E. McCready, and D. R. Baer, Journal of Applied Physics 94, 7926 (2003).

5. G. W. Zhou, Phys. Rev. B 81, 195440 (2010).

6. G. W. Zhou, J. C. Yang, Physical Review Letters 89, 106101 (2002).

7. G. W. Zhou, J. C. Yang, Applied Surface Science 210/3-4, 165 (2003).

8. G. W. Zhou, J. C. Yang, Surface Science 531/3, 359 (2003).

9. G. W. Zhou, J. C. Yang, Applied Surface Science 222, 357-364 (2004).

10. G. W. Zhou, J. C. Yang, Surface Science 559/2-3, 100-110 (2004).

11. Langli Luo, Yihong Kang, Zhenyu Liu, Judith C Yang, Guangwen Zhou, Phys. Rev. B, Accepted on March 2 $2^{\text {nd }}, 2011$

12. D. R. Gaskell, "Introduction to Metallurgical Thermodynamics" (Scripta, Washington, D.C., 1973). 